Claus Zittel

\title{
„Don Juan der Sprache“. Otokar Fischer und die tschechische Nietzsche-Rezeption
}

\section{Einleitung}

Über Jahrzehnte hatte sich Otokar Fischer intensiv mit Nietzsche auseinandergesetzt und wurde der wichtigste Vermittler von dessen Werk in Tschechien. ${ }^{1}$ Diese Vermittlung vollzog sich erstens anhand von Übersetzungen und Kommentaren, zweitens durch literaturwissenschaftliche Studien und drittens durch Rezensionen von deutschen und französischen Neuerscheinungen aus dem Bereich der Nietzsche-Forschung.

Im Kontrast dazu hat man Fischers Beiträge zu Nietzsche in der deutschen Nietzsche-Forschung bis heute weitgehend ignoriert, ${ }^{2}$ selbst dann, wenn sie in deutscher Sprache erschienen waren. Eine gewisse Ausnahme bildet Fischers Brieffreundschaft mit dem - im deutschen Sprachraum ebenfalls vollkommen wirkungslosen - selbsternannten Nietzsche-Nachfolger Rudolf Pannwitz ${ }^{3}$, von der zumindest ein Schlaglicht auf Fischers Stellungnahmen zum tschechisch-deutschen Kulturverhältnis fällt (Pannwitz 2002).

Im Folgenden werde ich versuchen, einen ersten Überblick über Fischers Nietzsche-Arbeiten zu geben, der jedoch vorläufig bleiben muss, da ich mich aufgrund meiner Unkenntnis der tschechischen Sprache auf

1 Im Nietzsche-Handbuch (Ottmann 2000) fehlt ein Eintrag zur tschechischen Nietzsche-Rezeption. Somit bleibt für die erste Phase gültig: Měšt'an (1985: 34-54). Zur philosophischen Rezeption in Tschechien siehe auch: Blecha (2007: 493-520) und Heftrich (1999).

2 Karl Jaspers führt Fischers Aufsatz über den Wiederkunftsgedanken lediglich als Beispiel für Sekundärliteratur zu Nietzsches Krankheit an. Jaspers (1936) und Michlová/Wolfová (1962) heben vor allem die persönliche Annäherung Fischers an Nietzsches Werk hervor, und betonen, er habe Nietzsche als Übergangsphänomen betrachtet: „Otokar Fischer sah in Nietzsche vor allem den Gelehrten, den Dichter, der den Einflüssen der Metaphysik und Realität ausgesetzt ist. Er hielt ihn für einen Menschen des Übergangs und des am RandeStehens“ (Michlová/Wolfová 1962: 83). Diese Einschätzung erscheint mir verfehlt.

3 Von Pannwitz hatte Fischer vor allem dessen Einführung in Nietzsche (1920), gelesen und diese Schrift mehrfach empfohlen. 
die deutschsprachigen Zeugnisse stützen muss. ${ }^{4}$ Diese sind allerdings zahlreich und prononciert genug, um zumindest einige wichtige Facetten und bemerkenswerte Eigentümlichkeiten von Fischers Nietzsche-Bild charakterisieren und bewerten zu können. Dabei wird sich zeigen, dass über die bloße Vermittlung von Texten und Gedanken hinaus, Fischers NietzscheBeiträge für die Fachgeschichte der Auslandsgermanistik von exemplarischer Bedeutung waren.

\section{Annäherungen}

Zunächst ist als umfangreichste Veröffentlichung zu Nietzsche Fischers frühe MonographieausdemJahr1913zunennen:FriedrichNietæsche.Literárnístudie. Kurs šstiprédnáskkový [Friedrich Nietzssche. Literarische Studie. Kurs in sechs Vorlesungen], die sich am üblichen Phasenmodell von Nietzsches philosophischer Entwicklung orientiert. Parallel zu den im ersten Kapitel skizzierten biographischen Stationen schreitet Fischer nach und nach das Werk ab. Die frühen Texte Nietzsches werden unter dem Titel „Nietzsches Romantik“5 verhandelt, gefolgt von einer Darstellung der mit Menschliches, Alliumenscbliches einsetzenden positivistischen Phase im Kapitel „Nietzsches Intellektualismus“, die Zarathustra-Zeit wird unter „Nietzsches Mystik“"verbucht. Jenseits von Gut und Böse und Zur Genealogie der Moral werden als Spätwerke vorgestellt, die als neue Themen biologistische Theorien einführten. Abschließend wird „Nietzsches Radikalismus“ anhand der letzten Schriften Der Fall Wagner, Der Antichrist und Götzendämmerung diskutiert und die Dionysos-Dithyramben sub specie der Frage, ob Nietzsche psychisch krank war, verhandelt.

Die 360 Seiten starke Gesamtdarstellung erschien vor Fischers Übersetzungen und bleibt als Einführung für Studenten in Vorlesungsform weitgehend konventionell. Bemerkenswert ist jedoch Fischers Versuch, sich mit literaturwissenschaftlichen Mitteln einen Zugang zu Nietzsche zu

4 Ich bin Michal Topor für seine Archivrecherchen zu großem Dank verpflichtet. Ohne seine Bereitstellung der Materialien hätte ich diesen Essay nicht schreiben können.

5 Das Inhaltsverzeichnis: 1. Životopis [Biographie]; 2. Nietzschova romantika [Nietzsches Romantik]; 3. Nietzschův intelektualismus [Nietzsches Intellektualismus]; 4. Nietzschova mystika [Nietzsches Mystik]; 5. Nietzschův směr k vědecko-filosofické soustavě [Nietzsches Tendenz zum wissenschaftlich-philosophischen System]; 6. Nietzschův radikalismus [Nietzsches Radikalismus]. 
verschaffen und den von diesem selbst veröffentlichten Schriften Priorität einzuräumen.

Allgemein gehalten sind auch die anhand von Nietzsches früher fragmentarischer Schrift Wir Pbilologen angestellten Betrachtungen zum Gegensatz von Kunst und Wissenschaft, die Fischer in seinem Aufsatz Über den Anteil des künstlerischen Instinkts an literarhistorischer Forschung einstreut (Fischer 1914: 102f.).

Es folgen Fischers Nietzsche-Übersetzungen, zuerst jene noch heute gültige von Also sprach Zarathustra. Diese mit knappem Kommentar versehene Ausgabe erlebte in der Zeit von 1914-1932 vier Auflagen (1914, 1920, 1925, 1932). In ihrem Erscheinungsjahr schwärmte Arne Novák in einem Aufsatz zu Nietæssche und die Tschechen: „Ohne übertreiben zu wollen wird man diesen ,Zarathustra' dem Schönsten zurechnen müssen, was die tschechische Übersetzungskunst aufzuweisen hat [...]" (Novák 1914: 1613-1616). ${ }^{6}$

1923 folgte die Übertragung von Die Geburt der Tragödie. Es ist also primär das im engeren Sinn ästhetische Werk Nietzsches, das Fischer ins Tschechische übersetzt.

1925 ist es Fischer selbst, der in einem Aufsatz für die Prager Presse ebenfalls über Nietssche und die Tschechen. Zum 25. Todestag Nietzsches ${ }^{7}$ schreibt. Er bilanziert, dass es erstaunlich sei, „wie willig sich unser Geistesleben von einem Genie befruchten ließ, dessen Grundtendenzen denjenigen der tschechischen Philosophie entgegensetzt waren." Kein Tscheche werde bei Nietzsche erwähnt, nicht einmal Comenius und Hus, und umgekehrt müsste man erwarten, dass das „Evangelium des Machtmenschen“, dass der „erdenfrohe Verkünder des Übermenschen“ im traditionell religiös

6 Novák zufolge setzte die Beschäftigung mit Nietzsche in Tschechien bereits vor 1900 bei dem neuromantischen Dichter Julius Zeyer, bei Karolina Světlá und Jaroslav Vrchlický ein. Aber „,begeistert und dankbar durfte sich zu Nietzsche als zu ihrem Lehrer und Befreier erst jene Generation bekennen, die seit den Neunzigerjahren der tschechischen Literatur das Gepräge gibt. Während es vor dem Abgrund des kulturbiologischen Problems der Dekadenz ratlos stand, begrüßte dies Geschlecht Nietzsches Mahnruf zum Lebenswillen, zur Macht, zur Tat, ließ sich von ihm zum positiven Individualismus erziehen, fand in ihm ein willkommenes Gegengewicht gegen die herrschenden kollektivistischen Tendenzen. Als Künstler half er die realistische Nüchternheit des Ausdrucks überwinden und die sprachmelodischen Werte schätzen [...]“ In diese „Schar von Nietzsches Schülern“ gehören „Arnošt Procházka, Jiř́ Karásek, F. V. Krejč̌́, F. X. Šalda, J. S. Machar und Otokar Březina, mit seinen Schriften setzen sich auseinander: V. Kalina, A. Podlaha, František Krejč́, Lev Borský und Otokar Fischer."

7 Fischer (1925b); siehe auch den Nachtrag in der Prager Presse vom 13.09.1925: Nietzsche in Böhmen (Fischer 1925c). 
orientierten Tschechien auf taube Ohren stoßen müsste. Jedoch sei es eine „überraschende Tatsache“, dass in Tschechien sowohl „stark sozial empfindende“ als auch „prononciert nationale Geister [...] zu Pionieren des Nietzschekults geworden sind." Fischer geht zunächst auf den sozialistischen Essayisten Krejč ein, der sogar den Willen zur Macht als Mitleidsphilosophie deute und dessen unzulängliche Übersetzungen dennoch zum ersten Mal in Nietzsches Dichtung philosophisches und künstlerisches Neuland für die Tschechen erschlossen. Hingegen feierte die um die Zeitschrift Moderní Revue gescharte Künstlergruppe Nietzsches vermeintlich aristokratische Fin-de-siècle-Stimmung, Nietzsche den Immoralisten, den „Meister der Invektive und der Paradoxe“. Es sei ein Widersinn des „tschechischen Nietzschekultes“, dass der „Gegner aller décadence von den Theoretikern der décadence auf das Schild" gehoben wurde. An den älteren Übersetzungen bemängelt Fischer, dass sie den „Geist der Schwere“ nicht haben überwinden können und das „tanzend Übermütige der Wortspiele und Wortwitze“ nicht adäquat wiederzugeben vermocht haben (Fischer 1925b). Übersetzt seien zwei der Unzeitgemässen Betrachtungen, die Geburt der Tragödie und der Zarathustra (Fischer verweist ohne seinen Namen zu nennen auf seine Übersetzung), Götz̧en-Dämmerung, Der Antichrist und Ecce homo, der eigentlich philosophische Nietzsche hingegen (Menscbliches Allzumenschliches, Die Morgenröthe, Die Fröbliche Wissenschaft, Zur Genealogie der Moral, Der Wille zur Macht) harre noch der Übersetzung und Einbürgerung.

Dennoch gäbe es eine gewichtige philosophische Rezeption. Fischer verweist auf Masaryk $^{8}$, der Nietzsches Übermenschenlehre polemisch in die Linie der Deszendenz-Selektionstheorien stelle und diverse andere Auseinandersetzungen. Von Seiten der Literaturwissenschaft sei 1913 dann sein eigener Beitrag hinzugetreten.

Das „Nachwirken des tiefdringenden Philosophen und grossen Stilisten“ (Fischer 1925b) sei indes mehr als in der Philosophie in der Literatur der Jahrhundertwende in Tschechien zu spüren:

Dem tschechischen Vers hat sich der Meister scharfen Pointierens des öfteren als melodischer und dialektischer Führer erwiesen, gewiss ist auch für die tschechische Prosa noch manche Belehrung und Anfeuerung durch den hohen Kenner der schriftstellerischen Nuance, den Feinschmecker und Ziseleur des Worts, den wißbegierigen, alle Hüllen durchleuchtenden Seelenrater, den Don Juan nicht bloss der Erkenntnis, sondern auch der Sprache, zu erwarten. (Fischer 1925b)

8 Dazu weiterführend: Heftrich (1998: 107-143) und Hrubý (1988). 
Auch diese Bilanz macht klar, dass sich Fischer hier noch primär für das ästhetische Wirken Nietzsches interessiert. Das soll sich bald ändern.

\section{Nietzsche und die deutsche Literaturgeschichte}

Bleiben wir aber zunächst noch im literarischen Beziehungsgeflecht. Fischer ging es um die Einordnung Nietzsches in die deutsche und europäische Literaturgeschichte. Im Nachlass erhalten ist ein Manuskript Zur literarischen Geschichte des deutschen Individualismus (Nietzsche) und offenbar sollte Nietzsche hier mit den entsprechenden Vorläufern in eine Reihe gestellt werden. Einen konkreten Eindruck, wie Fischer diese Linie zu ziehen gedachte, gibt sein 1911 und somit noch vor der Nietzsche-Monographie erschienener Aufsatz: Nietzsche und Kleist (Fischer 1911c: 506-519). ${ }^{9}$

Fischer erblickt zwischen Kleist und Nietzsche eine Verwandtschaft in der Radikalität und Erkenntnisskepsis, denn beide würden „den spitzigsten Stachel gegen den eigenen Geist richten, um als echte tragische Helden an sich selber zugrunde zu gehen“" (Fischer 1911c: 506). Doch sei nicht nur die Wesensverwandtschaft, sondern über Nietzsches Lehrer in Schulpforta, Koberstein, auch eine direkte Beeinflussung fassbar und diese sei prägend gewesen:

Dies ist nun gerade meine Überzeugung. Kleist war nicht bloß ein schöner Zufall in Nietzsches Leben - wie Stendhal, wie Dostojewski -[,] sondern Grundlage seiner Bildung. Die Spuren sind in Nietzsches Schreibart nachzuprüfen. (Fischer 1911c: 517)

Fischer führt - chronologisch Nietzsches Werke durchstreifend - Stellen an, in denen er ähnliche Gesinnungen erkennen will, die gleiche „Unrast“", das „Bis-ans-Ende-Gehen“ und das „Durchkosten einer Gemütsstimmung, bis daß sich der Rausch in Ekel wandelt" (Fischer 1911c: 507). Überhaupt scheine Nietzsches „Groll gegen das Übergewicht der Theorie [...] stark Kleistisch gefärbt" (Fischer 1911c: 512). Ausgehend von einem Nachlassnotat Nietzsches, in dem davon die Rede ist, dass „,die vollkommene Erkenntnis [...] das Handeln" (Fischer 1911c: 512) töte, werden Parallelen zu Kleists KantKrise gezogen und durch Zitate mit ähnlichem Tenor aus Kleists Schriften zu belegen versucht. Vorgenommen wird auch eine literaturgeschichtliche

9 Vgl. dazu den Beitrag von Dieter Heimböckel in diesem Band. 
Verortung: Nietzsches Antihistorismus sei seinerseits historisch bedingt, allgemein vorbereitet durch die Literaten des „Sturm und Drang“, der „Romantik“ und des „Jungen Deutschland“ (Fischer 1911c: 510f.), konkret in den Ansichten von Herder, Goethe, Ludolf Wienbarg und Heine.

Die Analogien wirken indes gesucht, und man muss sie als begrenzte und willkürliche Konstruktion möglichst zahlreicher Gemeinsamkeiten ansehen. ${ }^{10}$ Im Lichte der neueren Forschung erscheint Nietzsche klar und durchgehend als Anti-Romantiker, Kleist wird jedoch von den Romantik-Verdikten weitgehend ausgenommen - hier wäre also stärker zu differenzieren gewesen.

Festzuhalten ist jedoch Fischers Hinweis auf Wienbargs Ästhetische Feldzüge - diese Relation wurde in der Forschung erst viel später und ohne Hinweis auf die Priorität Fischers in der Forschung gewürdigt. ${ }^{11}$ Der Schluss des Essays ist allerdings bemerkenswert, denn Fischer erkennt nun doch auch das Trennende und schlägt bereits das apologetische Leitthema seiner späten Nietzsche-Texte an, wenn er Nietzsche nicht nur zwischen, sondern über den nationalen Kulturen positioniert:

Nietzsche hingegen ist von Sehnsucht nach einer kommenden Zeit erfüllt; in seinen Mythen vom Übermenschen und von der Wiederkunft greift er an Werte der Ewigkeit. Kleist, der stärkere Künstler, mutet wie ein unvergleichliches Drama des deutschen Geisteslebens an. Nietzsche jedoch, den Verächter der nationalen Schranken, wollen wir nicht verschweizert, nicht naumburgisch und thüringisch werden lassen, ihn reklamieren wir als europäisches Ereignis. (Fischer 1911c: 519) ${ }^{12}$

10 Das gilt auch für die ältere Darstellung Joels (1905), die ähnlich vorgegangen war. Siehe dazu meine Artikel „Romantik“ und „Kleist“ im Nietssche-Handbuch (2000: 315-317, 388f.).

11 Siehe: Ridle (1980: 338-355). Nach Fischer hat auch Alfred Kerr in seinem Vorwort zu Wienbarg (1919: 1-3), die Parallelen gesehen: „Wer spricht: Wienbarg oder Nietzsche? wenn es etwa heißt: "Das Leben ist des Lebens höchster Zweck« -? Der Satz steht in diesem Buch. Wer spricht, Wienbarg oder Nietzsche? wenn es heißt: »Befreit die Welt von den Sünden der Schwäche« -? Der Satz steht wieder in diesem Buch. (Auch der andre: die herrschende Moral stelle walles Treibende und Liebende in uns [...] als das Sündhafte dar«.) Ja, dergleichen ist zwar ein Vorklang für Nietzsche. Doch nicht minder, scheint mir, ein Nachklang: von der deutschen Romantik; von Friedrich Schlegel und dem ganz jungen Clemens Brentano. Nietzsche war ein wildgewordener Romantiker - das Junge Deutschland aber hat erkennenden Verstand auf die blaue Blume gepfropft. Auch Wienbarg. Es war kein Unglück."

12 Fischer greift hier ein Nietzsche-Wort auf, der in Götzen-Dämmerung geurteilt hatte: „Goethe - kein deutsches Ereignis[,] sondern ein europäisches“" (Nietzsche 1999: 49). 


\section{Déjà-vu und ,Ewige Wiederkunft}

In seinem Aufsatz über Das Problem der Erinnerung ${ }^{13}$ kommt Fischer auf Nietzsche am Rande zu sprechen (Fischer 1911a: 7); in einem zweiten Text zur Thematik, der nun allerdings heute noch überaus anregend, ja spektakulär ist, rückt er ihn ins Zentrum der Betrachtung: Drei Jahre vor Freuds Studie zur fausse reconaissance $(1914)^{14}$ veröffentlicht Fischer in der Zeitschrift für allgemeine Psychologie seine Überlegungen zum Phänomen des déjà-vu: Eine psychologische Grundlage des Wiederkunftsgedankens. Bemerkungen über den literarischen Wert der "fausse reconnaissance" (Fischer 1911b). ${ }^{15}$

In der Nietzsche-Forschung ist dieser Aufsatz nahezu vollkommen übersehen worden. Zwar erwähnt Richard Krummel ihn knapp in seinem Kompendium Nietzsche und der deutsche Geist ${ }^{16}$ doch das ging dort in der Flut der anderen 5600 abstracts unter. ${ }^{17}$ Es gibt in der Nietzsche-Forschung drei Hauptlesarten ${ }^{18}$ der Lehre oder genauer des Gedankens der Ewigen Wiederkunft des Gleichen: die primär auf Nachlassnotizen sich stützende kosmologische Deutung, die entweder mythisch oder naturwissenschaftlich begründet wird, und die ethische Interpretation. Den Gedanken der Ewigen Wiederkunft in Verbindung mit dem Déjà-vu zu bringen und primär an den Schreibverfahren der veröffentlichen Schriften Nietzsches zu explizieren, ist originell $^{19}$ und von Fischer selbst in den möglichen Konsequenzen gar nicht erkannt worden.

Sogenannte Erinnerungstäuschungen wie die fausse reconnaissance oder ihr Pendant, das pathologische Plagiat, besäßen neben ihrem „psychologisch-medizinischen Wert auch ein kunsttheoretisches, besonders literaturwissenschaftliches Interesse" (Fischer 1911b: 487). Ausgehend von dem längeren Zitat einer Beschreibung, mit der Henri Bergson solche

13 Vgl. dazu den Beitrag Manfred Weinbergs in diesem Band.

14 Freud (1914: 116-123). Zum déjà-vu als Phänomen siehe den Band: Oesterle (2003).

15 Später auf Tschechisch; Fischer (1929b).

16 Richard Frank Krummel: Nietzsche und der Deutsche Geist, Band 1 (umfasst die Jahre 18671900), 1974 (2. Auflage 1998); Band 2 (1901-1918), 1983 (2. Auflage 1998); Band 3 (19191945), 1998; Band 4: Ergänzungen, Berichtigungen und Gesamtverzeichnis, 2006.

17 Eine positive Würdigung von Fischers Aufsatz findet sich im ebenfalls unbeachtet gebliebenen Buch: Beckerhoff (1998: 29-37).

18 Nach wie vor einschlägig: Marton (1996: 42-63).

19 Außer bei dem auf Fischer zurückgreifenden Beckerhoff (s.o.) findet sich diese Idee sonst nur noch bei Hofmiller (1931: 74-131, hier: 74-79). 
Erinnerungsphänomene einzufangen versuchte, untersucht Fischer dann ähnliche Berichte in wissenschaftlichen und literarischen Texten (z. B. Charles Dickens, Friedrich Spielhagen, Heinrich Zschokke, Iwan Gontscharow und Arthur Schnitzler), und zeigt auf - und das ist nun wirklich methodisch höchst interessant-, dass es einen Zusammenhang von Beschreibungssprache und beschriebenem Gefühlseindruck gibt, mithin eine besondere Art und Weise, wie sich Erinnerungstäuschungen sprachlich manifestieren. Einem solchen Erlebnis gehe oft das Gefühl voraus: „Wache oder träume ich?“ Die Unsicherheit hierbei sei Anzeichen einer „Depersonalisation“ (Fischer 1911b: 493). Literaturpsychologisch könne man mit dieser Gefühlscharakteristik Dichtungen besser erfassen, die das Leben als Traum, Halbträume und Erinnerungsträumereien darstellen.

Die nächste Unsicherheit fände ihren typischen Ausdruck in der „ohnmächtigen Anstrengung des Gedächtnisses“ in der Vergangenheit bestimmte Ereignisse zu lokalisieren („das dumpfe Sich-besinnen und Sichnicht-besinnen-können"). Diese führe zu einer Rückprojektion aktueller Erlebnisse ,in einen Traum, in die Kindheit, in graue Vergangenheit [...] oder gar ein Vorleben. “ (Fischer 1911b: 498), wobei diese Rückprojektionen dazu dienen können, ansonsten rätselhafte Erlebnisse zu erklären. Dadurch komme auch „dem Gefühl der Übereinstimmung zwischen Gesichtseindruck und Innenwelt“ eine „poetische Bedeutung“ zu, das Erleben eines Déjàvu zähle daher zu den „künstlerisch fruchtbarsten Augenblicken“ (Fischer 1911b: 513).

Hiermit ist der Boden bereitet, um „eines der schwierigsten und lockendsten Probleme der modernen Metaphysik“ anzugehen, nämlich „eine literarpsychologische Analyse der Lehre Friedrich Nietzsches von der Ewigen Wiederkunft des Gleichen“ (Fischer 1911b: 501). Gerade die Wiederkunftslehre sei „eine crux interpretum“ für Nietzscheverehrer, denn manche, die sich zwar an der Übermenschvision erbauten, würden hier „,vom geliebten Hodegeten“ Abschied nehmen, andere „den mystischen Gedanken als bloße Spielerei“ abtun (Fischer 1911b: 501). Nietzsche aber habe „für den unglaubwürdigsten Artikel seiner Philosophie einen ganz bedingungslosen Glauben in Anspruch genommen und ihn als Grundlage seines Systems bezeichnet" und ihm im Zarathustra „poetische Weihe und prophetische Verklärung“" verliehen. Gerade angesichts des Positivismus der späteren Werke befremde dieser plötzliche mystisch-religiöse Rückschlag. Unterlag Nietzsche hier also „einem Selbstbetrug“? (Fischer 1911b: 502)

Fischer rekonstruiert dann auf der Basis der Nachlassnotate die „Wiederkunftstheorie“ (Fischer 1911b: 502ff.), bringt mögliche Vorläufer 
Rezeption

ins Spiel (Fischer 1911b: 506) und verweist dann auf eine Stelle (Zweite Unzeitgemässe Betrachtung) beim jungen Nietzsche, in der dieser bereits allerdings kritisch - den Wiederkunftsgedanken dargestellt habe. Entgegen der späteren Selbststilisierung Nietzsches, dass ihn dieser Gedanke in Gestalt einer plötzlichen Vision überfallen habe, war er ihm also bereits früher bekannt. Fischer analysiert die verschiedenen Schilderungen, die Nietzsche von diesem Erlebnis gegeben hat und bestimmt es als ein unbewusst gebliebenes Déjàvu-Gefühl (Fischer 1911b: 509).

Dafür jedoch, dass „zwischen Wiederkunftsgedanken und Erinnerungstäuschungen bei Nietzsche eine tatsächliche Beziehung stattgehabt" habe, meint Fischer ein positives Argument vorbringen zu können, nämlich wenn man just die Art und Weise untersuche, wie dieser Gedanke formuliert wurde. Zum Ausgangspunkt nimmt Fischer einen Passus aus einem Kapitel des dritten Zarathustra-Buches: „Vom Gesicht und Rätsel“, in dem „die Wiederkunftslehre wohl zum ersten Mal vor der Öffentlichkeit in zusammenhängender Form aufgerollt" sei, und zwar eingebettet in einer fortlaufenden Erzählung und ,in die Atmosphäre eines schwermütigen Traums getaucht, daher von symbolischer Sprache verschleiert" (Fischer 1911b: 509). Es folgt eine eingehende Traumanalyse, an deren Ende Fischer „eine Reihe typischer Züge festgehalten“ findet, „,denen wir bei der Besprechung literarisch verwerteter Erinnerungstäuschungen bereits begegnet sind“" (Fischer 1911b: 511). Etwa würden undeutliche oder Scheinerinnerungen an die Kindheit eingestreut, die Aufnahme von äußeren Geräuschen in den Traum geschildert, wie das Bellen eines Hundes, das wiederum als Quasi-Reminiszenz eine weitere Assoziationskette auslöst. Übertragen auf Nietzsches eigene Schilderung seiner Vision der Ewigen Wiederkunft werde erklärbar, ,daß sein unerweislicher Glaube an die Ewige Wiederkunft des Gleichen durch jene Zustände mit bedingt und zugleich gefärbt war, die ihm die Gegenwart als Wiederholung eines bereits erlebten Augenblicks vorspiegelten." (Fischer 1911b: 512)

Ich kann hier nicht ins Detail gehen, ${ }^{20}$ sondern will nur hervorheben, dass dies die erste und für lange Zeit einzige Interpretation der Ewigen Wiederkunft ist, die die konstitutive Bedeutung der literarischen Darstellungsform vorführt.

20 Siehe dazu Zittel (2015). 


\title{
5. Nietzsche als Politikum (Fischers Rezensionen und Beiträge für die Prager Presse)
}

\author{
Diese vorwiegend ästhetisch-psychologische Nietzsche-Lesart hat Fischer \\ jedoch durch eine zunehmend politisch ausgerichtete Betrachtung ergänzt, \\ wie ich abschließend anhand seiner für die Prager Presse verfassten Rezensionen \\ und Beiträge darstellen werde. \\ Die Rezensionen widmen sich hauptsächlich Veröffentlichungen aus dem \\ Kreis des Weimarer-Nietzsche-Archivs und der französischen Nietzsche- \\ Rezeption. ${ }^{21}$ Der letzte Punkt ist insofern bedeutsam, weil in Frankreich die \\ Formierung des Faches Germanistik eng mit dem Wirken zweier Nietzsche- \\ Spezialisten, Biographen und Übersetzer zusammenhing, Charles Andler ${ }^{22}$ \\ und Henri Lichtenberger ${ }^{23}$, die sich beide für ein positives Deutschlandbild
}

21 Daneben gibt es noch kurze Rezensionen: Fischer (1926b): kurze Rezension von August Vetters Nietzsche-Buch; Fischer (1929c) - Rezension der preisgekrönten Schrift: Fritz Krökel: Europas Selbstbestimmung durch Nietzsche (1929); Fischer (1931c): Paul Cohn: Nietzsches Untergang (durch Rauschmittel, von E. Förster-Nietzsche ebenfalls zur Erklärung des Spätwerks behauptet). Zur französischen Nietzsche-Rezeption siehe: Bianquis (1929), Le Rider (1997).

22 Andlers Hauptwerk: Nietzsche, sa vie et sa pensée (6 Bände). Paris: Bossard 1920-1931. Zu Andler gibt es gleich mehrere Rezensionen und einen Nachruf. Rezension Fischers (1926a) vom 03.08.1926: Charles Andler (1926): Nietzsche und Jakob Burckhardt (das ist eine Auskoppelung aus Bd. 1 von Andlers Nietzsche-Monographie). Fischer referiert zustimmend, dass Burckhards Entdeckung des agonalen Prinzips des Griechentums Einfluss auf die Kulturauffassung Nietzsches in der Geburt der Tragödie hatte, zudem Burckhards Andeutungen zum „Machtwillen“ in der griechischen Polis aufs spätere Werk Nietzsches eingewirkt hätten. Fischer (1931b): Rezension des 6. und letzten Bandes von Charles Andlers monumentaler Nietzsche-Darstellung, die sich abschließend dem Spätwerk zuwendet. Nietzsche wird hier als romantischer Enthusiast porträtiert, dessen Schwärmerei sich zunehmend in Skepsis verwandle. Ausführlich sei die Zarathustra-Mystik dargestellt und die literarischen Einflüsse extensiv behandelt, viele neue Quellen ins Spiel gebracht. Also sprach Zarathustra werde als großzügiges Fragment verstanden, dessen Fortsetzungspläne aus dem Nachlass rekonstruiert werden. Bedeutsam sei auch, was über Nietzsche als Vorläufer von Durkheim und Sorel gesagt werde. Leitend sei für die Darstellung die „Reziprozität von Denken und Erleben“.

23 Siehe Fischer (1929a): Kurze Anzeige der deutschen Übersetzung des Buches des französischen Germanisten Henri Lichtenberger: Nietzssche und sein Werk, gemeinsam mit Elisabeth Förster-Nietzsche veröffentlicht, die es übersetzte. Die Rezension gibt keinen Hinweis auf die länger vorliegende tschechische Übersetzung. Lichtenberger war von 1887 bis 1907 Professor für ausländische Literatur in Nancy, danach bis 1934 Professor für Literatur und deutsche Philologie an der Sorbonne. Er hatte den kranken Nietzsche 1898 noch persön- 
und die deutsch-französische Aussöhnung einsetzten und deren Bücher Fischer bespricht. Man kann sagen, dass Fischer in Tschechien eine ähnliche Rolle für die Fachgeschichte spielte, wie Andler in Frankreich. In dem Maße wie nun Nietzsche immer stärker in Deutschland für nationalistische Zwecke vereinnahmt wurde, bekommen Auslandsgermanisten, die sich mit Nietzsche für ihr Fach einsetzen, Legitimationsprobleme, die sich auch auf das ganze Fach erstrecken. Andler und Lichtenberger waren in ihrer Gesinnung sozialistisch ausgerichtet, dennoch arbeiteten sie lange eng mit dem NietzscheArchiv in Deutschland zusammen, das zunehmend die nationale Propaganda bediente, zugleich aber auch durch die Beförderung von Übersetzungen und gezielt selektiertem Informationsfluss die gesamteuropäische Wirkung Nietzsches zu verstärken und zu kontrollieren suchte. Auch Fischer hatte offenbar persönlichen Kontakt mit Elisabeth Förster-Nietzsche, jedenfalls beruft er sich auf mündliche Mitteilungen und lobt sie als ,liebevolle Walterin des brüderlichen Nachlasses“ (Fischer 1925a) und "verdienstvolle Frau“ (Fischer 1935: 139). Vor allem aber rezensiert er zunächst wohlwollend ihre werkbiographischen Deutungen ${ }^{24}$ und die vom Archiv besorgten Editionen, was ihn später zu Differenzierungen nötigen wird.

Unter anderem bespricht Fischer die von Nietzsches Cousin Max Oehler besorgte Ausgabe von Nietzsches Jugendschriften (Fischer 1923). Oehler war Mitarbeiter im Nietzsche-Archiv, dessen Leitung er nach Elisabeth Förster-Nietzsches Tod übernahm. Wie diese betrieb er die Eingemeindung Nietzsches ins Reich der NS-Ideologie. Fischer lobt hier zunächst die vom „rührigen Nietzsche-Archiv“ aus dem Nachlass des „Dichterphilosophen“ veranstaltete Edition des „Hauptwerkes“ Der Wille zur Macht. Er begrüßt, dass dieser Text, „aufgrund wiederholter Prüfung der Manuskriptbestände in erweiterter Gestalt und übersichtlicher Anordnung vorgelegt werden

lich aufgesucht. Auch seine frühere Nietzsche-Biographie war von Nietzsches Schwester Elisabeth Förster-Nietzsche, mit der er auch einen regen Briefwechsel führte, 1899 ins Deutsche übersetzt worden: La Philosophie de Nietzssche, Paris 1898; dt.: Die Philosophie Friedrich Nietzsches, Dresden 1899.

24 Siehe die Besprechung in der Prager Presse am 07.10.1923 (Fischer 1923) und 20.08.1925 (Fischer 1925b) von Der werdende Nietzsche: Autobiographische Aufzeichnungen. Hrsg. von Elisabeth Förster-Nietzsche (München: Musarion-Verlag, 1924), die dann in einen Vergleich Nietzsches mit Hebbel übergeht. Immerhin auch hier die Einschränkung, dass die „knabenhaften Aufzeichnungen des noch frommen Nietzsche durchaus nicht überschätzt werden“ dürften. Im Vergleich mit Hebbel zeigen sich die Grenzen biographischer Darstellung, bei dem „wandlungsreichen und widerspruchsvollen“ Nietzsche lasse sich nicht von einer Epoche seines Lebens auf die nächste schließen, kein Werdegang genetisch explizieren. 
konnte" (Fischer 1923: 1). Hingegen sei die Edition der Jugendschriften aufgrund ihrer chronologischen Ordnung problematisch, da nun Parerga und Fragmente neben ausgereiften und auskristallisierten Werken zu stehen kommen, statt im Anhang gebracht zu werden. Fischer sieht die Vorzüge, die diese Ausgabe Spezialisten bietet, doch er rät zur Vorsicht, denn wenn jemand mit dieser Ausgabe Nietzsche zu lesen beginne, würde er zuerst mit den „faden Kindereien“ seiner „Gymnasiastenpoesie“ konfrontiert und nicht - wie von Nietzsche selbst gewünscht - mit der die Geisteswelt des Philosophen eröffnenden „Offenbarung“ (Fischer 1923: 1) des Gegensatzes von Dionysischem und Apollinischem in der Geburt der Tragödie.

Immerhin kann man in diesem Plädoyer für das veröffentliche Werk Nietzsches eine erste Distanzierung Fischers von den Nachlasseditionen erkennen, auf deren Konto auch die Fabrikation der berüchtigten Ideologeme von Nietzsches Philosophie gehen, denn in den von Nietzsche veröffentlichten Schriften sucht man vergebens nach ungebrochen positiv formulierten Lehren vom Übermenschen oder vom Willen zur Macht. ${ }^{25}$

Man sollte hier erwähnen, dass nicht erst Schlechta, sondern bereits Ernst und August Horneffer 1906 und 1907 die Unhaltbarkeit der Willen-zurMacht-Kompilation nachgewiesen hatten, allerdings nicht ohne Nietzsche als Philosoph herabzusetzen, dem die Kraft zum System gefehlt habe - womit sie auf heftigen Widerstand seitens der Nietzsche-Gemeinde trafen (Fuchs 1997: 384-404; Montinari 1982) -, ja auch Schlechtas fulminante Kritik wurde viele Jahre später noch attackiert und hier tat sich durch eine heftige Polemik, die 1957 im Merkurveröffentlicht wurde, Otokar Fischers Brieffreund Rudolf Pannwitz (1957) hervor. Die Konstellation Pannwitz-Fischer ist daher brisant, zum einen weil Pannwitz einen europäischen, transnationalen Nietzsche forderte, zum anderen, weil er die Nachlass-Fiktionen des Weimarer Archivs noch bis zum letzten Atemzug verteidigte. ${ }^{26}$

25 Der Tenor der Kritik lautet, dass das nicht veröffentlichte Werk als sekundär gegenüber den publizierten Schriften anzusehen ist (!). Die Jugendlyrik wird dann vorgestellt, aber nicht als selbstständig bedeutsam gewürdigt, sondern nur insofern als in ihr die spätere Dichtkunst (wie z. B. der ,herrliche Zarathustra-Hymnus, Vor Sonnenaufgang“") präludiere. Frühe Texte, wie das Euphorion-Fragment, das die neuere Nietzsche-Forschung wegen seiner radikalen Selbstreflexivität hoch einschätzt, werden in ihrer Bedeutung nicht erkannt. Gleichwohl lässt sich Fischer weit auf das Jugendwerk Nietzsches ein.

26 Pannwitz hatte bereits in seiner Schrift Geist der Tschechen (1919) die tschechische Kultur zum Vorbild für ein transnationales Europa erhoben. Die tschechische Kultur und Nietzsches Philosophie beförderten in seinen Augen das gleiche Ideal. Fischer empfahl Pannwitz' Einführung in Nietzsche Kollegen und in Vorträgen, konnte ihm aber nicht helfen, als 
1931 wird Fischer mit einem ziemlich üblen Propagandabuch, nämlich mit Theodor Kappsteins Edition eines „Volks-Nietzsche“ konfrontiert (Fischer 1931a), und übt heftige Kritik an der dort vorgenommenen Reinigung des Textes von Fremdwörtern und Satzzeichen, der freien Zusammenstellung von Texten mit neuen Titeln wie: „Morgenröte fröhlicher Wissenschaft“, und an der ,kastigierenden Bearbeitung“.

Dann aber wird der Rezensent Fischer (1932) mit dem wichtigen Buch von Erich Podach: Gestalten um Nietzsche (1932) konfrontiert. Dieses Werk muss ihm die Augen geöffnet haben, denn es ist der Hauptangriff gegen das Nietzsche-Archiv und die ,von dessen Leiterin ausgehende offizielle Nietzscheliteratur". Franziska Nietzsche, Erwin Rohde, Peter Gast und das Ehepaar Förster-Nietzsche werden in ihrem Gegensatz zu Nietzsche dargestellt, letztere aufgrund ihres Antisemitismus. Fischers Fazit: „Ein herbes Buch, das u.a. mit Bäumlers und Wolters Nietzsche-Kult abrechnet und das gewiss alles eher verdient als totgeschwiegen und von oben herab behandelt zu werden." ${ }^{\text {"27 }}$

Es folgt im Jahr darauf ein Nachruf auf Charles Andler (Fischer 1933), in dem deutlichere Töne angeschlagen werden. Fischer würdigt Andler als „führenden Germanisten Frankreichs“, lobt dessen „,edle sozialistische Gesinnung“ sowie dessen Überzeugung, dass „ein wachsam objektives Urteil über deutsche Angelegenheiten“ im „ureigensten Interesse Frankreichs" sei (und meint natürlich auch im Interesse Tschechiens). Insbesondere Andlers Charakteristik Nietzsches als „übernationales Phänomen und europäisches Ereignis“" sei hervorzuheben. ${ }^{28}$ Andler lasse Nietzsche, wie Goethe und Schiller, an dem „Hauptfehler der Deutschen“, deren ,inculture politique“ und am „Mangel des Verständnisses für wahre Demokratie“ teilnehmen. Andler

er aufgrund seiner Kritik an der tschechischen Außenpolitik Schwierigkeiten bekam. Siehe dazu die Einleitung von Marie-Odile Thirouin zum Briefwechsel Pannwitz-Fischer.

27 Interessant für einen Vergleich ist die ebenfalls durch Podachs Buch im gleichen Jahr mitausgelöste Kritik Walter Benjamins (Benjamin 1932) am Weimarer Archiv.

28 Siehe auch Fischers Brief an Pannwitz vom 09.03.1923, in welchem er seinen Vortrag Nietzsche und die Slawen bereits so zusammenfasst: „[M] ir war es darum zu tun, Nietzsche als europäisches Ereignis hinzustellen und ihn als einen Erzieher zu einer übernationalen Zukunft zu interpretieren." (Pannwitz 1923: 173) Ohne Zweifel wollte Fischer hiermit auf Distanz zu Richard Moritz Meyers Nietzsche-Bild gehen. Meyer, einer der Lehrer Fischers, hatte im Vorwort zu seinem Buch Nietzsche. Sein Leben und seine Werke (1913) als Ziel formuliert: „, $[\mathrm{N}]$ ach Philosophen, Theologen, Ästhetikern möchte auch der deutsche Philolog seinen Teil dazu beitragen, daß Nietzsche als ,deutsches Ereignis' angesehen und gewürdigt werde.“ (Meyer 1913, VIIf.). Zu Meyer siehe weiterführend: Fiebig/Waldmann (2009), zu Pannwitz und Nietzsche: Szabó (2015, 75-106). 
hebe die Wesensverschiedenheiten zwischen Deutschen und Franzosen hervor und wolle doch zur Völkerverständigung beitragen, wobei er sich

zum geistigvollem Schwung erhebt, wo es gilt, das germanisch-mystische Halb-Dunkel zu kontrastieren mit französischer Intellektualität, will sagen, mit eisigem grauen Frühlicht, aus dem ganz allmählich die Sonne emporsteigt. (Fischer 1933)

Diesen Gedankengang nimmt Fischer dann zum Anlass, Nietzsches Verhältnis zum Nationalsozialismus genauer zu klären und zwar in dem wichtigen Aufsatz Nietzsche und das dritte Reich, der 1934 auf Tschechisch, dann ein Jahr später in der Übersetzung von Johannes Urzidil in Die drei Ringe (Fischer 1935: 137-141) $)^{29}$ erschien.

Es handelt sich um eine der frühesten und differenziertesten Auseinandersetzungen mit der auch heute noch diskutierten Frage, ${ }^{30}$ inwieweit Nietzsche die NS-Ideologie vorbereitet hatte. „Haben die Ideologen des Dritten Reiches das Recht, sich auf Nietzsche als ihrem unmittelbaren Vorgänger zu berufen?", fragt Fischer gleich zu Beginn und antwortet:

Ja, sie haben das Recht, denn eine Menge von Aphorismen und Fragmenten, welche ein Regime der starken Hand empfehlen, gegen Verfallserscheinungen gerichtet sind, den Willen zur Macht zum wesentlichsten menschlichen Prinzip erheben, für die Rückkehr zum Primitivismus eifern [...], tragen tatsächlich ein Gepräge, das ohne Veränderung in den Rechts- und Sittenkodex der heutigen Herren Deutschlands übernommen werden könnte. (Fischer 1935: 137)

Man könnte jedoch „mit gleicher Berechtigung“ eine „Menge anderer Stellen“ zitieren, ,nach denen die Praxis der Hitlerleute nichtbloß als Karikatur, sondern als das direkte Gegenteil dessen erschiene", was Nietzsche intendierte (Fischer 1935: 137). Die Sache sei also komplex und bedürfe eingehender Betrachtung, für die er lediglich einige Richtlinien vorgeben könne. So verkomplizierten sich bei Nietzsche durch ,seine dichterische Ausdrucksform“ und Symbolik, durch das „schwankende und vieldeutige Wesen einzelner Passagen im Zarathustra" (Fischer 1935: 137) die Zusammenhänge weit mehr als bei anderen Philosophen. Nietzsches Urteile seien kaum je eindeutig zu greifen, wandelten sich zudem radikal „,von Extrem zu Extrem“ (Fischer 1935: 138).

29 Das Verbandsorgan der deutschen Freimaurer in der ČSR, Die drei Ringe erschien von 1925-1935 in Reichenberg [Liberec] und danach in Prag; Johannes Urzidil, damals selbst Freimaurer, hatte zwischen 1934-1938 dessen Schriftleitung inne.

30 Zum Beispiel Taureck 2000: 169-206. Eine frühe Stellungnahme, die Nietzsches Unvereinbarkeit mit dem Nationalsozialismus hervorhob und die Fischer womöglich 1934 als Vortrag gehört hatte, ist: Löwith 1987 [1934]. 
Rezeption

Dies habe auch zu den gegensätzlichen Deutungen seines Werkes geführt. In Böhmen habe der Nietzsche-Kult mit F. V. Krejč eingesetzt und widersprüchliche Züge angenommen, zeigte sich als Mitleidsphilosophie, künstlerischer Amoralismus (Procházka), Weltbürgertum (Šalda) oder konsequenter Nationalismus (Lev Borský). Ähnlich sei es im Ausland. In Italien sei jedoch über D'Annunzio und den Vorkriegsfuturismus eine direkte Verbindung zwischen Nietzsche und der faschistischen Ideologie festzustellen, in Deutschland seien es Moeller van den Bruck, Rosenberg, aber auch Stefan George und sein Kreis (Ernst Bertram, Friedrich Gundolf, Ludwig Klages) und Oswald Spengler gewesen, die in diesem Sinne Nietzsche auslegten. Hitler jedoch sei von Nietzsche unabhängig, seine Ideologie käme ganz ohne ihn aus, für sie genüge der Wiener Antisemitismus und NachWagnerianische Rassismus. Doch wurde der Nährboden für die Aufnahme solcher Anschauungen im Zeichen Nietzsches vorbereitet: „Um den Lyriker Stefan George schloß sich ein Kreis von Vergötterern und Interpreten, welche das Heil der Welt darin suchen, daß irgendein Mächtiger kommt und sich der unseligen Herde annimmt.“ (Fischer 1935: 139)

Gundolfs Schriften zur Literaturgeschichte seien mit ihren aristokratischen Wertmaßstäben in dieser Tradition zu sehen, ebenso Ludwig Klages, der Nietzsches „Antirationalismus“ zu Ende gedacht habe (Fischer 1935: 139).

Dann wendet sich Fischer Nietzsches Umgebung zu. Wie Ernst Podach kritisiert er, dass Paul Ree, August Julius Langbehn, Max Oehler, und die Försters im Widerspruch zu Nietzsche gestanden und seine Gedanken „simplifiziert und entseelt“ (Fischer 1935: 139) haben. „Die Tragik des heutigen Nietzscheismus“ liege darin, dass der „echte Nietzsche mit einem Mann verwechselt wird, den er nicht vertrug und dessen Tendenzen ihm auf die Nerven gingen“ (Fischer 1935: 139). Die Gefahr dabei sei, „daß Nietzsches Lehre zu einem engstirnig nationalistischen Evangelium reduziert würde, zu einer Verherrlichung der rein germanischen Tugenden und zu anderen Ideen, aus denen die jetzigen Machthaber Vorteile ziehen könnten“ (Fischer 1935: 139). Es bestünde daher die

Pflicht der unparteiischen Nietzsche-Interpretation [...] darin, den authentischen Nietzsche vor jenen zu schützen, die sich auf eigene Faust mit ihm identifizieren, ohne daß man sie mehr desavouieren könnte und die unter den Vertretern der offiziellen Nietzsche-ArchivForschung eine Stütze finden. (Fischer 1935: 139)

Es werden dann mit eindrucksvoller Klarheit weitere Pro- und Contra-Belege für die Vorläufer-These diskutiert:

Es fällt mir nicht etwa ein, den Beweis führen zu wollen, daß Nietzsche kein unmittelbarer Vorgänger der Bewegung gewesen wäre, die nach dem Kriege in Deutschland entstand 
und nach dem Jahre 1930 das Feld beherrschte. Die grossartigen Visionen, in den der späte Nietzsche seinen Blick auf die politischen und Massenkämpfe kommender Jahrhunderte heftet; die Lehre vom Willen zur Macht als Schlüssel alles menschlichen Handelns; der wütende Kampf gegen alles, worin er ,decadence' witterte; der Nachdruck, den er auf die Würde der Rasse[,] auf Reinblütigkeit, auf die Notwendigkeit des Krieges, auf die Raubtierinstinkte legt; das Symbol der blonden Bestie, das Ideal korsischer Banditen, die Sehnsucht nach dem Losbrechen geheimer Dämonen, die materialistische Auffassung religiöser und gedanklicher Emotionen, die physiologische Ausdeutung der Künste - diese und andere Grundsätze der letzten [...] Schriften Nietzsches bieten direkten Ansporn zu Gewaltsamkeiten, eine Anleitung zu anti-sozialistischen, antidemokratischen, fortschrittsfeindlichen Methoden. Der Versuch des Nachweises, die Etappe der gegenwärtigen innerstaatlichen Kämpfe und Diktaturen sei von Nietzsche nicht vorausgesehen und vorbereitet worden, hieße einen Mohren weiß waschen wollen. Freilich handelt es sich um Sätze, die einen einheitlich ausgeprägten Gesichtspunkt nicht zulassen, viele Antithesen aufweisen und deren Prophezeiungen in den Mantel der Vieldeutigkeit gehüllt erscheinen. (Fischer 1935: 140)

Es sei jedoch „sicher, daß die konkrete Verwirklichung der allgemeinen von Nietzsche vorausgesehenen Kämpfe eine Richtung genommen hat, die niemals seine Zustimmung gefunden hätte“ (Fischer 1935: 140). Fischer erinnert an Nietzsches Vision des ,guten Europäers“ und an sein Bekenntnis „Europa will eins werden“, die in scharfem Widerspruch zu „nationalistischem Wahn" stehen (Fischer 1935: 140). Insbesondere in Bezug auf den Antisemitismus klaffe „ein unübersteigbarer Abgrund“ (Fischer 1935: 140) zwischen Nietzsche und seinen heutigen Nachfolgern. Auch wenn Nietzsche kein Philosemit gewesen sei, so war er unzweifelhaft ein Anti-Antisemit. Fischers hellsichtiger Aufsatz ist noch nicht seine letzte Stellungnahme zu Nietzsche. In einer ebenfalls in der Prager Presse veröffentlichten Glosse zu einem Nietzsche-Zitat resümiert Fischer:

Ein jedes Jahrzehnt und ein jedes Volk formt sich seinen Nietzsche neu, und liest aus ihm heraus, was eben den eignen Anschauungen und Zielen am nächsten liegt. Kein Wunder also, daß im heutigen Reich eben solche Stellen am willigsten gehört [...] werden, die durch Betonung des nunmehr so zeitgemäßen und vielempfohlenen Irrationalismus an die Theorie und Praxis der Zeitgenossen von Fichte und Arndt unmittelbar anknüpfen. (Fischer 1937)

Man kann Fischer auch heute nur beipflichten. Sein prononciert ästhetischer Zugang zu Nietzsche erlaubte es ihm, dessen Sätze nicht einsinnig zu lesen und, als es darauf ankam, sie früh jenseits von Alfred Bäumler und Georg Lukács politisch differenziert zu beurteilen, - was zugleich die beste Strategie war, um in dieser Zeit das eigene Fach zu verteidigen. Die Besonderheit von Fischers Nietzsche-Lektüre besteht allerdings auch darin, dass er an Nietzsches Schreib- und Denkweise stärker die klassischen Züge hervorhob 
„Don Juan der Sprache“. Otokar Fischer und die tschechische Nietzsche-

Rezeption

und nirgends auf seine Bedeutung für die aktuelle Literatur der Avantgarde und der Moderne, insbesondere jener in Tschechien, ${ }^{31}$ näher einging.

31 Mit den Autoren der Prager Moderne stand Fischer in regem Austausch. Vgl.: Zittel 2016. 


\section{Literatur}

Andler, Charles (1920): Nietzsche, sa vie et sa pensée. Paris: Bossard.

Beckerhoff, Ulf (1998): Der Verlust der Aisthesis. Nietzsches Gedanke von der ewigen Wiederkehr des Gleichen aus Sicht seiner späten Philosophie. Marburg: Tectum-Verlag.

Benjamin, Walter (1932): Nietzsche und das Archiv seiner Schwester. - In: Die literarische Welt, 77-78. (Wiederabgedruckt in: ders.: Gesammelte Schriften. Bd. 3. Frankfurt/M.: Suhrkamp 1972, 323-326).

Bertram, Ernst (1918): Nietzsche. Versuch einer Mythologie. Berlin: Georg Bondi.

Bianquis, Geneviève (1929): Nietzsche en France. L' influence de Nietzsche sur la pensée française. Paris: Alcan.

Blecha, Ivan (2007): Nietzsche in der tschechischen Phänomenologie. Patočka und die Frage nach dem Sinn. - In: Studia Phaenomenologica 7, 493-520.

Fiebig, Nils/Waldmann, Friederike (Hgg.) (2009): Richard M. Meyer-Germanist zwischen Goethe, Nietzsche und George. Göttingen: Wallstein Verlag.

Fischer, Otokar (1911a): Das Problem der Erinnerung und deren Bedeutung für die Poesie, 4. Congresso Internazionale di Filosofia, Bologna. - In: Zeitschrift für Angewandte Psychologie und psychologische Sammelforschung. Leipzig: Barth, 1-8.

Fischer, Otokar (1911b): Eine psychologische Grundlage des Wiederkunftsgedankens. Bemerkungen über den literarischen Wert der „fausse reconnaissance“. - In: $Z A P$. Bd. 5, 487-515.

Fischer, Otokar (1911c): Nietzsche und Kleist. - In: Neue Jabrbücher für das Klassische Altertum. Bd. 27. Leipzig: Teubner, 506-519.

Fischer, Otokar (1914): Über den Anteil des künstlerischen Instinkts an literarhistorischer Forschung. - In: Zeitschrift für Ästhetik und allgemeine Kunstwissenschaft, 96-108.

Fischer, Otokar (1923): Nietzsches Jugendschriften. - In: Prager Presse 3, Nr. 275 (07.10.), Beilage Dichtung und Welt, Nr. 38, 1-3.

Fischer, Otokar (1925a): Zwei Werdegänge. - In: Prager Presse 5, Nr 227 (20.08.), 6.

Fischer, Otokar (1925b): Nietzsche und die Tschechen. Zum 25. Todestag Nietzsches. - In: Prager Presse 5, Nr. 232 (25.08.), 6.

Fischer, Otokar (1925c): Nietzsche in Böhmen. - In: Prager Presse 5, Nr. 251 (13.09.), $9 f$.

Fischer, Otokar (1926a): Charles Andler: Nietzsche und Jacob Burkhardt. - In: Prager Presse 6, Nr. 210 (03.08.), 6.

Fischer, Otokar (1926b): Nietzsche. Von August Vetter. - In: Prager Presse 6, Nr. 225 (18.08.), 6.

Fischer, Otokar (1929a): Nietzsche und sein Werk. - In: Prager Presse 9, Nr. 15 (15.01.), 7.

Fischer, Otokar (1929b): Déjà vu. - In: ders., Duše a slovo. Praha: Melantrich, 140-160. 
„Don Juan der Sprache“. Otokar Fischer und die tschechische Nietzsche-

Rezeption

Fischer, Otokar (1929c): Nietzsche und Frankreich. - In: Prager Presse 9, Nr. 207 (01.08.), 7.

Fischer, Otokar (1931a): Volksnietzsche? - In: Prager Presse 11, Nr. 118 (30.04.), 8.

Fischer, Otokar(1931b):VonCharles AndlersmonumentalerNietzschemonographie... - In: Prager Presse 11, Nr. 223 (19.08.), 7.

Fischer, Otokar (1931c): Paul Kohn: Um Nietzsches Untergang. - In: Prager Presse 11, Nr. 229 (25.08.), 7.

Fischer, Otokar (1932): Gestalten um Nietzsche. - In: Prager Presse 12, Nr. 187, (10.07.), Beilage Dichtung und Welt, Nr. 28, 3.

Fischer, Otokar (1933): Charles Andler. - In: Prager Presse 13, Nr. 96 (07.04.), 6.

Fischer, Otokar (1935): Nietzsche und das Dritte Reich. - In: Drei Ringe 11/9, 137 141.

Fischer, Otokar (1937): Glosse zu einem Nietzschezitat. - In: Prager Presse 17, Nr. 222 (14.08.), 6.

Förster-Nietzsche, Elisabeth (Hg.) (1924): Der werdende Nietzsche: Autobiographische Aufzeichnungen. München: Musarion-Verlag.

Förster-Nietzsche, Elisabeth/ Lichtenberger, Henri (1928): Nietzsche und sein Werk. Dresden: Reissner.

Freud, Sigmund (1914): Über fausse reconnaissance („déjà raconté") während der psychoanalytischen Arbeit. - In: ders., GW X, Frankfurt/M.: Fischer, 116-123.

Fuchs, Dieter (1997): Der Wille zur Macht: Die Geburt des „Hauptwerks“ aus dem Geiste des Nietzsche-Archivs. - In: Nietzsche-Studien 26, 384-404.

Heftrich, Urs (1999): Nietzsche v Céchách [Nietzssche in Böhmen]. Prag: Hynek.

Heftrich, Urs (1998): The Early Czech Nietzsche Reception: T.G. Masaryk, O. Březina, F.X. Šalda. Translated by H. Steilberg. - In: Freifeld, Alice/Bergmann, Peter/Glatzer Rosenthal, Bernice (Hgg.), East Europe Reads Nietzsche. New York: Columbia University Press, 107-143.

Hofmiller, Josef (1931): Nietzsche - In: Süddeutsche Monatshefte 29/2 (November), 74131.

Hrubý, Karel (1988): Masaryk and Nietzsche. - In: Novák, Josef (Hg.), On Masaryle. Amsterdam: Rodopi, 247-268.

Jaspers, Karl (1936): Nietzsche. Einführung in das Verständnis seines Philosophierens. Berlin: de Gruyter.

Joel, Karl (1905): Nietzsche und die Romantik. Jena/Leipzig: Diederichs.

Krummel, Richard Frank (1974): Nietzsche und der Deutsche Geist. Berlin: de Gruyter.

Le Rider, Jacques (1997): Nietzsche in Frankreich. München: Fink.

Löwith, Karl (1987 [1934]): Nietzsche der Philosoph unserer Zeit. - In: Sämtliche Schriften VI: Nietzsche. Stuttgart: Metzler.

Marton, Scarlett (1996): L'eternel retour du meme: these cosmologique ou imperatif ethique? - In: Nietzsche-Studien 25, 42-63. 
Měšt'an, Antonín (1985): Die erste Nietzsche-Rezeption bei den Polen und Tschechen. - In: Nietzsche kontrovers. Bd. 5. Würzburg: Königshausen und Neumann, 34-54.

Meyer, Richard (1913): Nietrsche. Sein Leben und seine Werke. München: Beck.

Michlová, Milada/Wolfová, Brigitta (1962): Das germanistische Werk Otokar Fischers. - In: Acta Universitatis Carolinae, Germanistica Pragensia II., 79-92.

Montinari, Mazzino (1982): Nietzsche zwischen Alfred Baeumler und Georg Lukács. - In: ders. (Hg.), Nietzsche lesen. Berlin: de Gruyter, 169-206.

Nietzsche, Friedrich (1999): Götzendämmerung. KSA 6. München: dtv.

Novák, Arne (1914): Nietzsche und die Tschechen. - In: Das literarische Echo 16, H. 23 (1. 9. 1914), Sp. 1613-1616.

Oesterle, Günter (2003): Déjà-vu in Literatur und bildender Kunst. München: Fink.

Ottmann, Henning (2000): Nietzsche-Handbuch. Stuttgart: Metzler.

Pannwitz, Rudolf (1920): Einführung in Nietssche. München: Hans Carl.

Pannwitz, Rudolf (1957): Nietzsche-Philologie? Rezension zu: Karl Schlechta: Friedrich Nietzsche, Werke in drei Bänden. - In: Merkur 11, 117, 1073-1087.

Pannwitz, Rudolf (2002): Briefwechsel mit Otokar Fischer und Paul Eisner. 1917-1936, hg. von Marie-Odile Thirouin. Stuttgart: Klett-Cotta.

Podach, Ernst (1932): Gestalten um Nietrsche. Weimar: Erich Lichtenstein Verlag.

Ridle, Hugh (1980): Nietzsche and Wienbarg: a consideration of parallels between Nietzsche and the young Germans. - In: Nietzsche-Studien. Bd. 9. Berlin/New York: de Gruyter, 338-355.

Szabó, László V. (2015): Renascimentum europaeum: Studien zu Rudolf Pannwitr. Berlin: Frank \& Timme.

Taureck, Bernhard H. F. (2000): Nietzsche und der Faschismus. Ein Politikum. Leipzig: Reclam.

Wienbarg, Ludolf (1919): Aesthetische Feldrüge. Hamburg/Berlin: Hoffmann \& Campe.

Zittel, Claus (2015): „Nachtwandler des Tages“. Traumpoetik und Parodie in Nietzsches Also sprach Zarathustra. - In: Pelloni, Gabriella/Schiffermüller, Isolde (Hgg.): Pathos, Parodie und Kryptomnesie. Das Gedächtnis der Literatur in Nietzsches ,Also sprach Zarathustra". Heidelberg: Winter, 125-169.

Zittel, Claus (2016): Poetik der Verschwommenheit. Philosophische, psychologische und ästhetische Wahrnehmungskonzepte in der Prager Moderne. - In: Stiegler, Bernd/ Werner, Sylwia (Hgg.): Laboratorien der Moderne. Orte und Räume des Wissens in Mittel- und Osteuropa. München: 2016, 49-95. 
„Don Juan der Sprache“. Otokar Fischer und die tschechische Nietzsche-

Rezeption 